\title{
Morphology of Bicuspid Aortic Valve in Adult Human Cadaver: In Correlation with Clinical Relevance
}

\author{
Kumar A ${ }^{1 *}$, Lal Ajmani $\mathbf{M}^{2}$ and Klinkhachorn PS $^{3}$ \\ ${ }^{1}$ Assistant Professor, Department of Anatomy and Neurobiology, Oman Medical \\ College, Oman \\ 2Professor and Head of Department, Department of Anatomy and Neurobiology, Oman
} Medical College, Oman

3Professor, Department of Pathology, Anatomy and Laboratory Medicine, West Virginia University Health Sciences Center, US

*Corresponding author: Dr. Anil Kumar, Assistant Professor, Department of structural anatomy and Neurobiology, PO Box 391, PC 321, Al-Tareef Sohar, Sultanate of Oman, Tel: + 968 22314761, + 968 9539 3982; E-mail: anil@omc.edu.om

\section{Abstract}

The bicuspid aortic valve (BAV) is the most frequent congenital anomaly of the heart in adults. BAV is composed of two cusps, morphologically and functionally. BAV are often associated with other cardiovascular malformations, coarctation of aorta, aortic dilatation. BAV is associated with number of complications; aortic stenosis, aortic regurgitation, aortic dissection and infective endocarditis. The morphology of the bicuspid or bicommissural aortic valve (BAV) may predict the severity of valve dysfunction. The aim of this study was to determine the relationship between aortic valve morphology in relation with its clinical significance. Sixty formalin fixed cadaveric hearts specimens irrespective of age, sex, and race were collected and serially numbered from 1 to 60 . The aortic valve of heart was dissected and opened. The characteristics and morphology of the bicuspid aortic valve is studied. A total of 3 specimens (5\%) had congenitally malformed valves (bicuspid valve) in 60 heart specimens examined. On dissection a well defined bicuspid aortic valve was found with calcification and friable valve leaflet. No other congenital anomalies were noted in these hearts. Analysis of BAV morphology is of clinical and prognostic relevance.

Keywords: Bicuspid Aortic Valve; Bicommissural Aortic Valve; Coarctation of Aorta; Aortic Dilatation; Endocarditis

Abbreviations: BAV: Bicuspid Aortic Valve; OMC: Oman Medical College

\section{Introduction}

Bicuspid aortic valve (BAV) is a common congenital cardiac malformation affecting $1-2 \%$ of the population with strong male predominance. It is likely that the presence of a bicuspid aortic valve has a genetic basis, with the pattern of transmission in some families suggesting an autosomal dominant pattern of inheritance [1]. The prevalence of BAV according to sex has been found to be 7.1 cases per 1000 among male neonates, and 1.9 per 1000 among female neonates [2]. The usual arrangement of the aortic valve is for it to have three 
leaflets; these are termed the right and left coronary leaflets and the non-coronary leaflet. Bicuspid aortic valve (BAV) describes as two of the three leaflets are formed and there is evidence of a raphe between the conjoined leaflets that control blood flow through the heart. Bicuspid aortic valves are often associated with coarctation of the aorta, aortic stenosis, aortic regurgitation, aortic dissection, aortic dilatation and infective endocarditis.

Individuals may have a normally functioning BAV and may be unaware of its presence and the potential risk of impending complications [3]. They may typically remain asymptomatic until the third or fourth decade of life, when the valve becomes dysfunctional. They then require close follow-up and valve replacement may be warranted [4]. It is now accepted that BAV is associated with both valve disease and aortic disease, thereby leading to increased morbidity and mortality, including aortic valve disorders, aortic wall abnormalities, endocarditis and other cardiovascular malformations [2].

The recognition of the bicuspid valve in patients with aortic valve disease remains an important challenge to the clinician, whereas preoperative knowledge of valve morphology would be helpful in planning the surgery. The study or case report mentioned in most of the literature about these embryological remnants was based on echocardiographic findings. There is paucity of literature about the anatomical study of these structures. It also possesses diagnostic confusion since it mimics other pathological manifestations. Hence, the aim of this study is to find the incidence of these rare embryological remnants with its morphology and possible associated clinical manifestations.

\section{Material and Methods}

The present observational descriptive study was conducted at the department of Anatomy and Neurobiology, Oman Medical College, Sohar, Sultanate of Oman. The specimens were provided by West Virginia University Health Sciences Center, Morgantown, WV. In this study, total sixty formalin fixed cadaveric heart specimens irrespective of age, sex, and race were collected for the purpose of teaching undergraduate students were utilized. The specimens which were putrefied, showed crush and cut injury of heart specimens were excluded from the study. Ethical clearance from Institutional Ethical Committee was obtained. The specimens were serially numbered from 1 to 60 . The Aortic valve of heart was opened and dissected. The characteristics and morphology of the bicuspid aortic valve is studied.

\section{Result and Discussion}

Fusion of the right and left valve cushions at the beginning of valvulogenesis appears to be a key factor in BAV formation. The fewer the number of cusps, the greater is the chance that the valve is stenotic from birth [5]. In the present study, BAV has been noticed in a 3(5\%) specimen out of 60 hearts studied. Bicuspid aortic valve is the commonest etiology of aortic stenosis between the ages of 60 and 75 years (59\% of cases); it was also the cause in $40 \%$ of those aged under 60 years and $32 \%$ of those aged more than 75 years [6]. The incidence of aortic stenosis complicating BAV in an autopsy series ranges from $15 \%$ to $75 \%$. The anatomy of the bicuspid valve may also influence the propensity for obstruction: Stenosis is more rapid if the aortic cusps are asymmetrical or in the antero-posterior position [4]. Progression of BAV stenosis is age related, with fibrosis beginning in the second decade and calcification progressing significantly after the fourth decade [7].

The presence of a BAV is an independent risk factor for progressive aortic dilation, aneurysm formation, and dissection. Aortic root dilatation is common in bicuspid aortic valve, even when the valve is haemodynamically normal. Apoptosis may be one of the mechanisms responsible for aortic medial smooth muscle cell loss associated with aortic dilatation and aneurysm formation in the setting of BAV disease [8]. Aortic regurgitation occurs in the presence of a BAV usually from cusp prolapse, fibrotic retraction, or dilation of the sinutubular junction. Infective endocarditis is responsible for between $43 \%$ and $60 \%$ of cases of severe aortic regurgitation in patients with bicuspid aortic valve, the result of cusp perforation in the majority of cases [9]. The incidence of bicuspid aortic valve is $1-2 \%$ and that serious complications occur in at least one third of cases, this condition may be responsible for more deaths and morbidity than the combined effects of all other congenital heart defects [10].

There is a wide spectrum of BAVs with variability in appearance and morphology. Ward C (2000) reported three characteristics of bicuspid aortic valve: inequality of cusp size, the presence of a central raphe (ridge), usually in the centre of the larger of the two cusps, and smooth cusp margins even in diseased valves [4]. In the present study, one of the specimens showed the free margins of both cusps were relatively linear in appearance, or the margins of one of the 2 cusps had a V-shaped configuration, with the apex of the $\mathrm{V}$ pointing toward a raphe (Figure 1). Other specimen shows, the free margins of the raphe cusp were concave, and the free margins of the non-raphe cusp were convex, such that the valvular 
orifices were nevertheless usually competent. The ventricular aspect of the valve beneath the raphe in all instances was flat or nearly so. Furthermore, on the aortic aspect at the site of the raphe, the $\mathrm{V}$ shaped cuspal margins were not visible in the raphe (Figure 2). On surgical resection in another specimen showed, a well defined bicuspid aortic valve was found with calcification and friable valve leaflet (Figure 3). The stenotic valves were obstructed by nodular, calcareous masses, but commissural fusion with diffuse calcification of valve leaflet is supported by adhesion. The evidence for attributing calcification and other deposits on the aortic aspects of the cusps would be due to patients with homozygous familial hypercholesterolemia which develops into aortic stenosis [11]. Tutar, et al. (2005) reported that aortic leaflet calcification starts as a focal area on the aortic side of the leaflet with subendothelial accumulation of lipoproteins and an inflammatory cell infiltrate [2]. BAVs may progress and become calcified, thus leading to varying degrees of severity of aortic stenosis or aortic regurgitation, or both, which may eventually necessitate surgical intervention [12]. Ward C (2000) suggested that in pediatric patients, $70 \%$ to $85 \%$ of stenotic aortic valves are bicuspid, and at least $50 \%$ of adults with aortic stenosis have BAV. This may reflect a propensity to premature fibrosis, and calcification [4]. Collins, et al. (2008) found that among the BAV patients included in their study, calcification of the base, calcification of the commissure, and ossification were found in $39.1 \%, 43.3 \%$, and $7 \%$ of the valves [13]. Lamas and Eykyn (2000) found that among $50 \mathrm{BAV}$ patients with infective endocarditis, $86 \%$ had anemia with a mean hemoglobin level of $11.8 \pm 1.58 \mathrm{~g} / \mathrm{dL}$. Anemia can be a result of several disorders of the aorta and aortic valve [14].

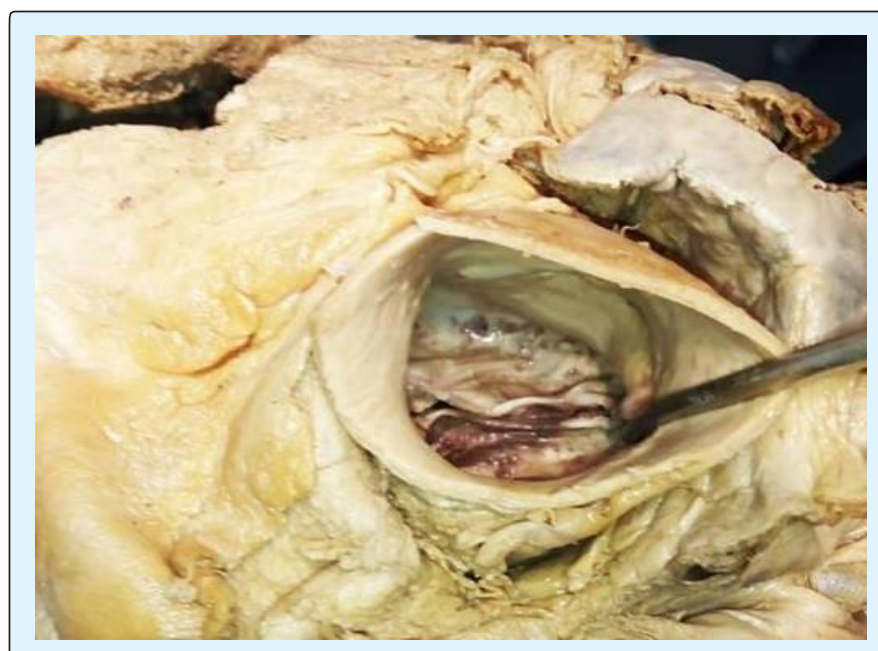

Figure 1: Picture of bicuspid aortic valve where both cusps are linear in appearance.

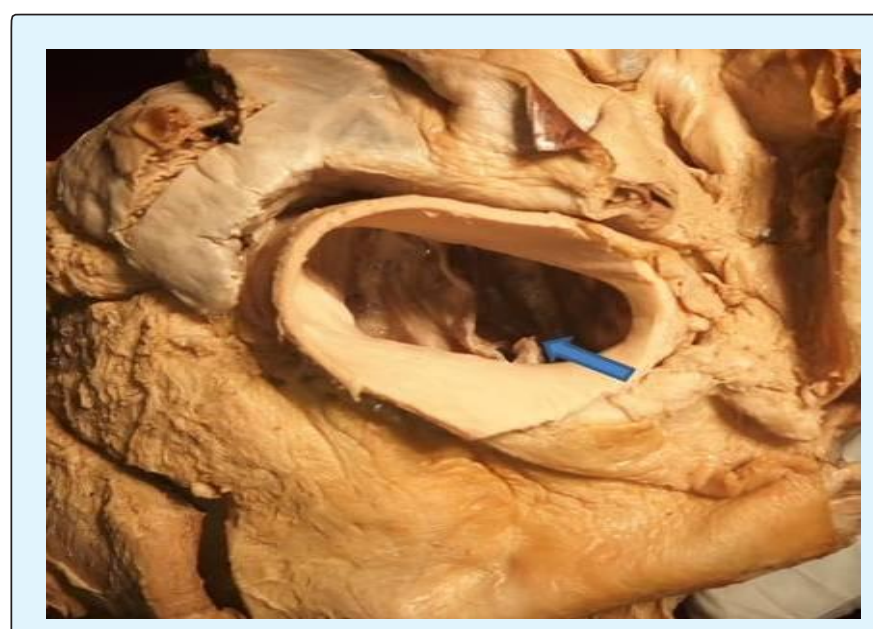

Figure 2: Picture of bicuspid aortic where V-shaped cuspal margins are not visible in the raphe (arrow).

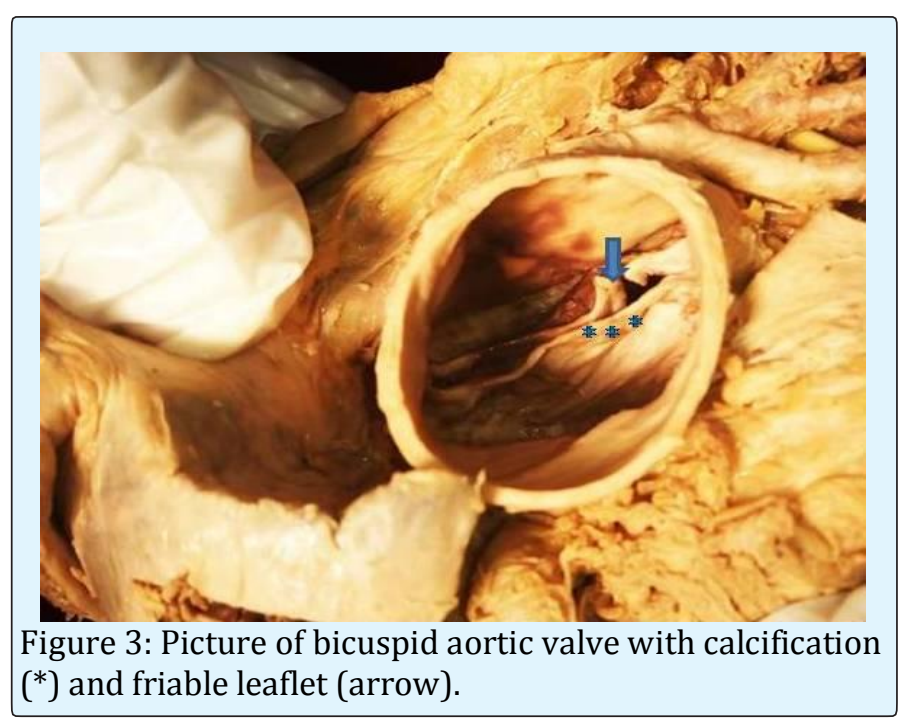

Scholz, et al. reported that $24 \%$ of the BAV patients in their study had a left dominant cardiac circulation [15]. Hutchins, et al. found a higher incidence of left dominance, $29 \%$, in postmortem angiography among the patients with the BAVs in their study [16]. It has been recognized that the BAV demonstrates abnormal leaflet motion, including folding or wrinkling of the valve tissue and increased leaflet doming during the cardiac cycle. This may result in increased currents of turbulence even when the leaflets are not stenotic [8]. Collins, et al. reported a prevalence of infective endocarditis of $1.5 \%$ among patients with the BAVs in his study, while we observed a prevalence of $4.11 \%$ [13].

The BAV, appearing on gross examination as an aortic valve with two cusps, has clinical relevance and has 
gained increasing interest in the medical community from a pathogenetic and especially surgical point of view.

\section{Conclusion}

The recognition of the bicuspid valve in patients with aortic valve disease remains an important challenge to the clinician, whereas preoperative knowledge of valve morphology would be helpful in planning the surgery. To confirm the results of this study, a prospective study with a larger sample size and a matched control group should be performed.

\section{Acknowledgments}

The authors would like to acknowledge the support exerted by Dr. M. L. Ajmani, Professor and Head, department of Anatomy and Neurobiology, Oman Medical College (OMC), Oman. The authors submit their sincere thanks to Dr. Penprapa S. Klinkhachorn, Professor of Department of Pathology, Anatomy and Laboratory Medicine, West Virginia University for providing the specimens. The authors extend their gratitude to Dr. Saleh Al Khusaiby, Dean, Dr. Mohammad Al Shafaee, Vice Dean, and Dr. Mubarak Pasha, Deputy Dean of OMC. The authors also thank the faculty and technicians of OMC for their help to complete this study.

\section{References}

1. Clementi M, Notari L, Borghi A, Tenconi R (1996) Familial congenital bicuspid aortic valve: a disorder of uncertain inheritance. Am J Med Genet 24(62): 336-338.

2. Tutar E, Ekici F, Atalay S, Nacar N (2005) The prevalence of bicuspid aortic valve in newborns by echocardiographic screening. Am heart J 150(3): 513515.

3. Lewin MB, Otto CM (2005) The bicuspid aortic valve: adverse outcomes from infancy to old age. Circulation 111(7): 832-834.

4. Ward C (2000) Clinical significance of the bicuspid aortic valve. Heart 83(1): 81-85.

5. Fernandez B, Duran AC, Fernández-Gallego T (2009) Bicuspid aortic valves with different spatial orientations of the leaflets are distinct etiological entities. J am coll cardiol 54(24): 2312-2318.
6. Pomerance A (1972) Pathogenesis of aortic stenosis and its relation to age. Br Heart J 34(6): 569-574.

7. Tadros TM, Klein MD, Shapira OM (2009) Ascending aortic dilatation associated with bicuspid aortic valve: Pathophysiology, molecular biology, and clinical implications. Circulation 119(6): 880-890.

8. Braverman AC, Beardslee MA (2009) The bicuspid aortic valve. In: Otto C, Bonow R (Eds.), Valvular heart disease: a companion to Braunwald's heart disease. Philadelphia: Saunders/Elsevier, pp: 169-186.

9. Murphy ES, Rosch J, Rahimtoola S (1977) The frequency and significance of coronary arterial dominance in isolated aortic stenosis. Am J Cardiol 39(4): 505-509.

10. Nistri S, Sorbo MD, Marin M, Palisi M, Scognamiglio R, et al. (1999) Aortic root dilatation in young men with normally functioning bicuspid aortic valves. Heart 82(1): 19-22.

11. Sprecher DL, Schaefer EJ, Kent KM, Gregg RE, Zech LA, et al. (1984) Cardiovascular features of homozygous familial hypercholesterolemia: analysis of 16 patients. Am J Cardiol 54(1): 20-30.

12. Fenoglio JR, Mcallister HAJR, Decastro CM, Davia JE, Cheitlin MD (1977) Congenital bicuspid aortic valve after age 20. Am J Cardiol 39 (2): 164-169.

13. Collins MJ, Butany J, Borger MA, Strauss BH, David TE (2008) Implications of a congenitally abnormal valve: a study of 1025 consecutively excised aortic valves. J Clin Pathol 61(4): 530-536.

14. Lamas CC, Eykyn SJ (2000) Bicuspid aortic valve-A silent danger: analysis of 50 cases of infective endocarditis. Clin Infect Dis 30(2): 336-341.

15. Scholz DG, Lynch JA, Willerscheidt AB, Sharma RK, Edwards JE (1980) Coronary arterial dominance associated with congenital bicuspid aortic valve. Arch Pathol Lab Med 104(8): 417-418.

16. Hutchins GM, Nazarian IH, Bulkley BH (1978) Association of left dominant coronary arterial system with congenital bicuspid aortic valve. Am J Cardiol 42(1): 57-59. 